\title{
Harmonic signal synthesis system design based on STC15 + TLC5620
}

\author{
Huaqun Zhan ${ }^{a}$, Yonghao Zhou ${ }^{b}$, Huarong Chen ${ }^{c}$ \\ Jiangxi normal university of science and technology, Nanchang 330013, China \\ a772815060@qq.com, b313473168@qq.com, 'c15979099109@qq.com
}

Keywords: STC15W1K24S, harmonic signal, signal synthesis.

\begin{abstract}
Analyze the signal synthesis process on Matlab theory platform, based on the theory STC15W1K24S chip and 4 road 8 digit serial d/a TLC5620 produced by TI Company are designed to be a signal synthesis circuit system. By using the system module we can created the one, three, five, seven time's harmonic component of the signal, those signal components could be compounded to the original signal. The harmonic components and the synthetic wave of any number of times can be observed and analyzed by means of an oscilloscope. The system can realize the synthesis of any four signals, and it is a portable waveform synthesize, which can be used to simply observe and analyses of signal synthesis in laboratory and outdoor.
\end{abstract}

\section{Introduction}

Any signal are made of different frequency, amplitude and initial phase of sine wave. As the periodic signal, its harmonic frequency is integer times of the fundamental by fourier expansion. As non-periodic signal, it contains all the frequency components from zero to infinity. Signal synthesis is an important topic of the signal field need to study, We often use MATLAB tools to study on virtual signal synthesis, But this kind of realizing signal synthesis on the virtual platform is the result of the ideal state, While ignoring the real problem during the process of synthesis, In view of this situation, this paper introduces a kind of method whose main control chip is STC15W1K24S, Control TLC5620 synthesize harmonic signal generated by STC15W1K24S into square wave signal.

\section{Signal synthesis principle analysis}

Anyone with a cycle of $\mathrm{T}$ wave function $\mathrm{f}(\mathrm{t})$ can be expressed as a series of trigonometric function of the sum, namely:

$$
f(t)=\frac{1}{2} a_{0}+\sum_{n=1}^{\infty}\left(a_{n} \cos n \omega t+b_{n} \sin n \omega t\right)
$$

Among them: $\mathrm{T}$ for cycle, $\omega$ for angular frequency, $\omega=\frac{2 \pi}{T}$, the first item $\frac{a_{0}}{2}$ for the dc component.

Fourier decomposition of the periodic function is dividing periodic function into dc component and all $\mathrm{n}$ order harmonic compositions.

As shown in figure 1 of the method can be written as:

$$
f(t)=\left\{\begin{array}{cc}
h & \left(0 \leq t<\frac{T}{2}\right) \\
-h & \left(-\frac{T}{2} \leq t<0\right)
\end{array}\right.
$$

The square wave is odd function, it has no constant term. Math can prove this square wave can be represented as:

$$
f(t)=\frac{4 h}{\pi}\left(\sin \omega t+\frac{1}{3} \sin 3 \omega t+\frac{1}{5} \sin 5 \omega t+\frac{1}{7} \sin 7 \omega t+\cdots \cdots\right)
$$




\section{3. signal synthesis theory analysis on Matlab}

clc; clear all;\% remove all of the variables in the workspace $\mathrm{x}=0: 1: 105$; \% variable assignment by colon $\mathrm{x}$

a=uint8(hex2dec(\{'7F','87','8E','96','9D','A5','AC','B3','BA','C0','C7','CD','D3','D8','DE','E3','E7,'E B','EF','F3','F6','F8','FA','FC','FD','FE','FE','FE','FE','FD','FB','F9','F7','F4','F1','ED','E9','E5','E0','DB','

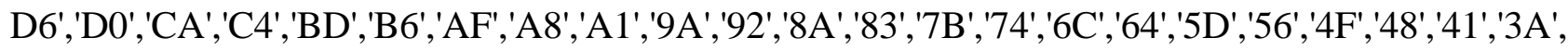

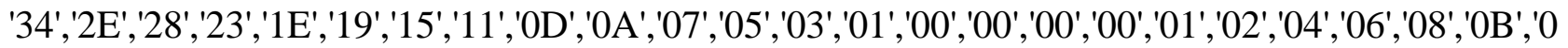
F','13','17','1B','20','26','2B','31','37','3E','44','4B','52','59','61','68','70','77','7F'\}));\% Convert hex to decimal give to Matlab functions for reading

Figure (1); \% open the graphics window

Plot $(\mathrm{x}, \mathrm{a}) ; \%$ complete the drawing of a harmonic

According to this step in order to complete three times, five times, seven times harmonic signal graphics drawing
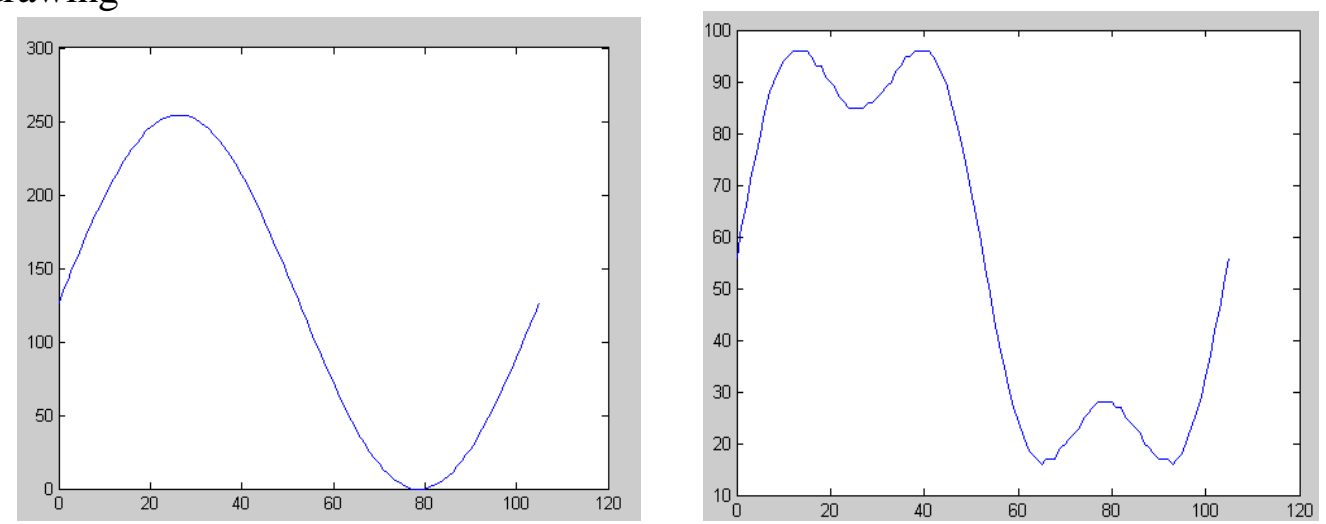

Fig. 1 A harmonic

Fig. 2 One, three times harmonic synthesis
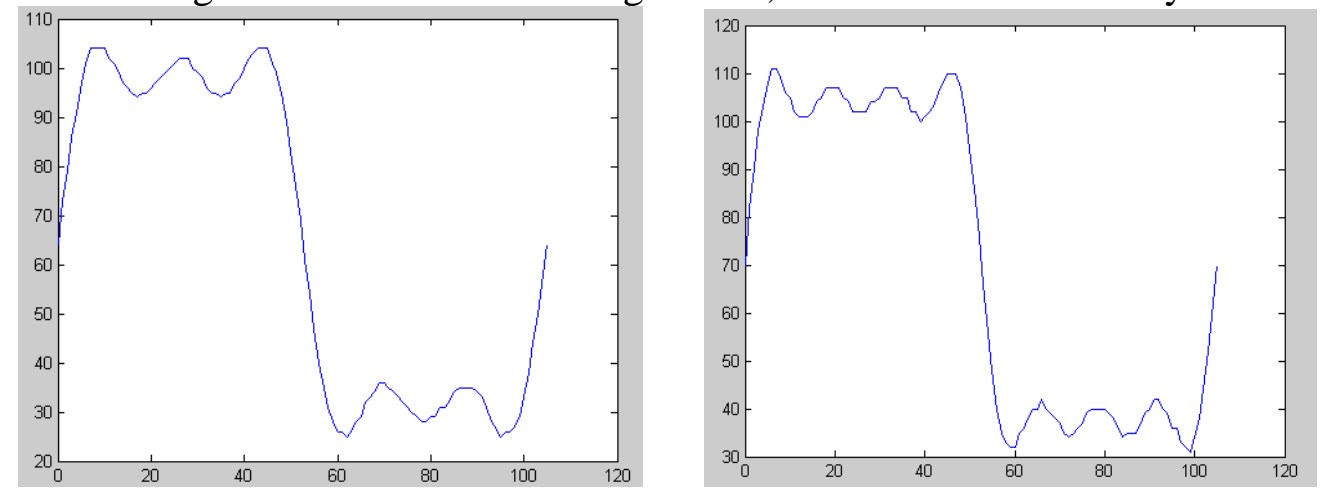

Fig. 3 One, three, five times harmonic synthesis

Fig. 4 One, three, five, seven times harmonic synthesis

\section{Hardware platform to realize signal synthesis}

\section{1 system overall scheme design}

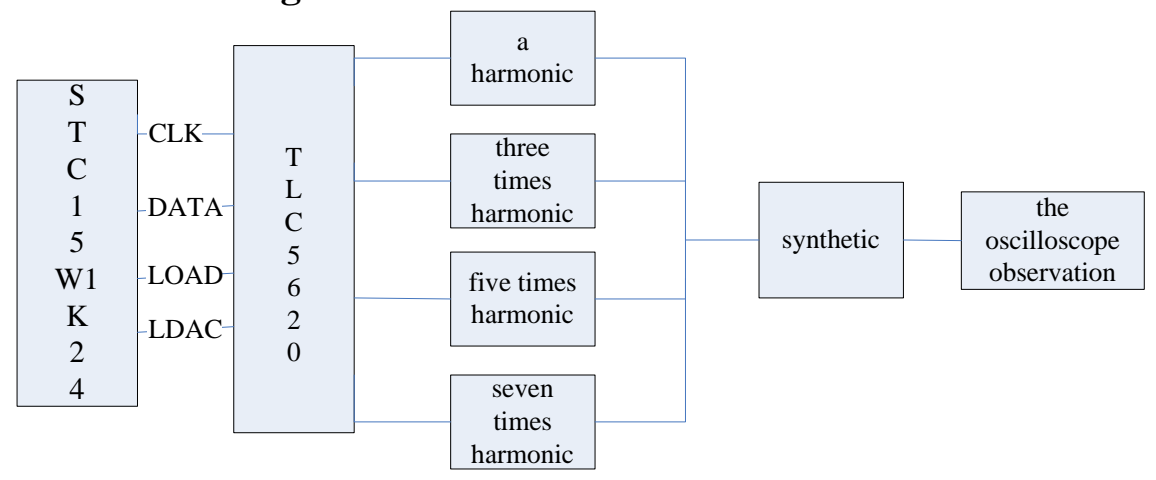

Fig. 5 System diagram 
This design will connect MCU's four I/O with the CLK ,DATA, LOAD, LDAC of the TLC5620 to drive it work, fundamental wave, three times harmonic, five times harmonic, seven times harmonic produced by the TLC5620 can travel through the op-amp realize harmonic signal synthesis. The oscilloscope can be used respectively to observe the fundamental wave, every harmonic and composite wave.

\section{2 control circuit part}

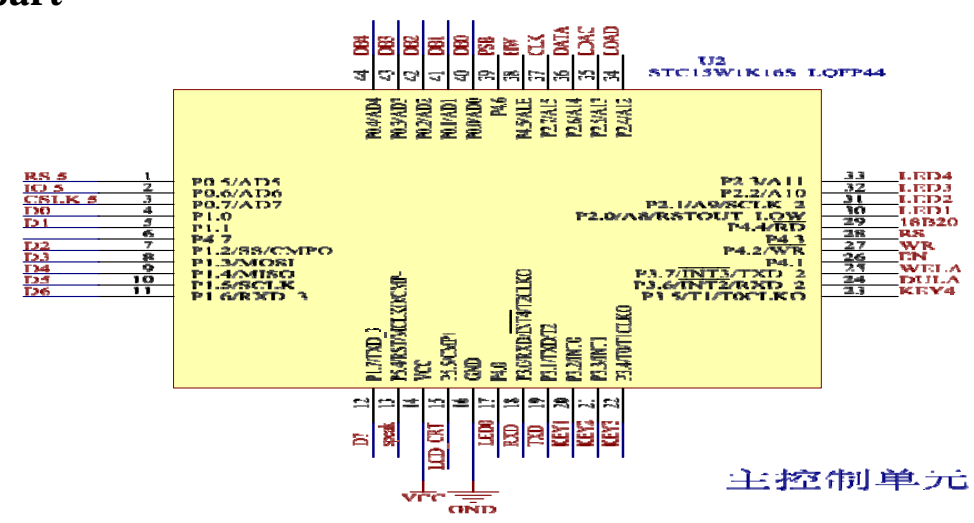

Fig. 6 Control circuit part

The system uses STC15W1K24S as the main control chip, which is a single clock cycle/machine single chip microcomputer produced by STC company with wide voltage/reliable high speed/low power consumption, strong anti-interference. Hold the STC ninth encryption technology, unable to decrypt, instruction code is fully compatible with traditional 8051, but 8 to 12 times as fast. ISP programming $5 \mathrm{MHZ}$ - $35 \mathrm{MHZ}$ wide range can be set up, can eliminate external expensive crystals and external reset circuit. Now hold STC15 series microcontroller using STC - Y5 ultra-high speed CPU cores, under the same clock frequency, faster than early $1 \mathrm{~T}$ series single-chip computer by $20 \%$. The DATA, CLK, LOAD, LDAC can realize serial communication with TLC5620.

\section{3 the harmonic signal generate circuit parts}
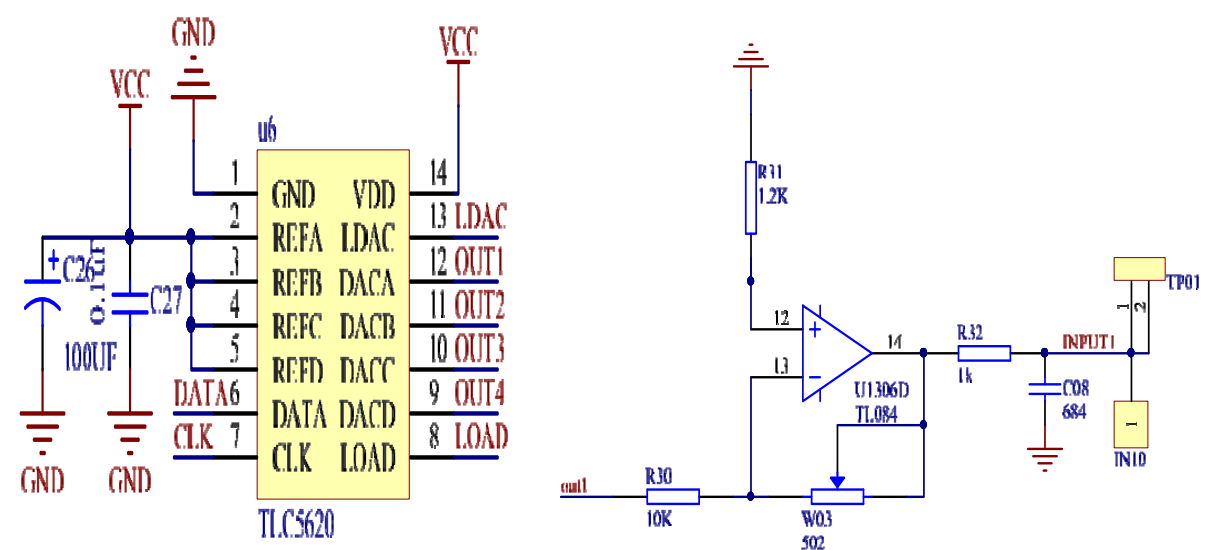

Fig. 7 Signal decomposition filter circuit

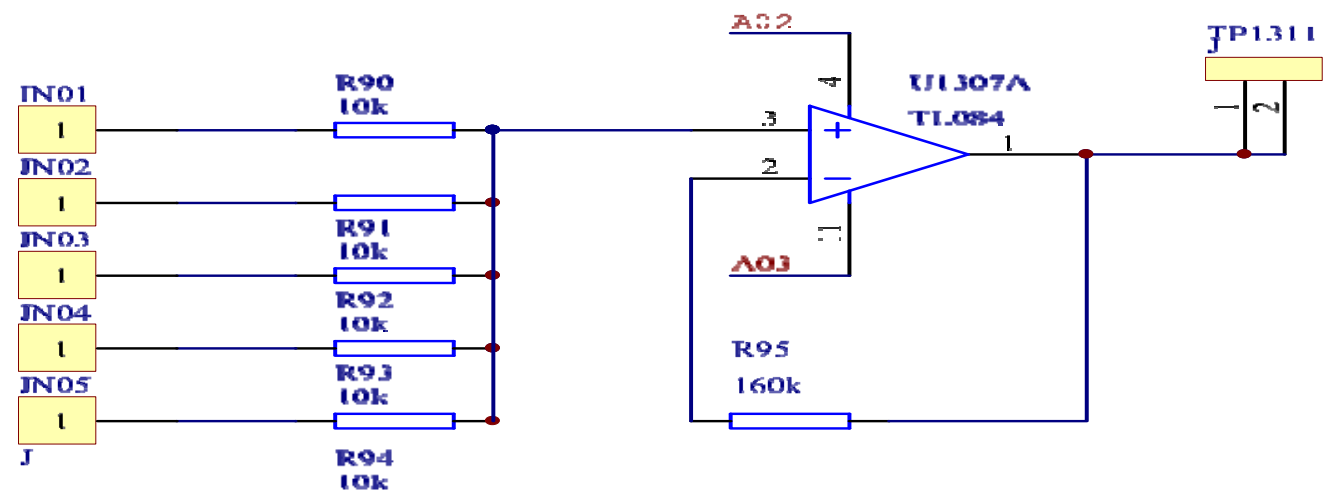

Fig. 8 Harmonic synthesis section 
TLC5620 through the DATA, CLK, LOAD, LDAC communicate with single chip microcomputer. C26, C27 stability feed reference voltage into DACA, DACB, DACC, DACD. Four road transformed harmonic signal output through the operational amplifier filtering. The output amplitude of the harmonic signal can be changed by the sliding rheostat. Use an oscilloscope can observe various harmonic signals.

\section{4 harmonic synthesis section}

One, three, five, seven harmonics can be synthesized by an adder, Thus synthesis the square wave form, which can be observed through the oscilloscope.

\section{5 system test results}
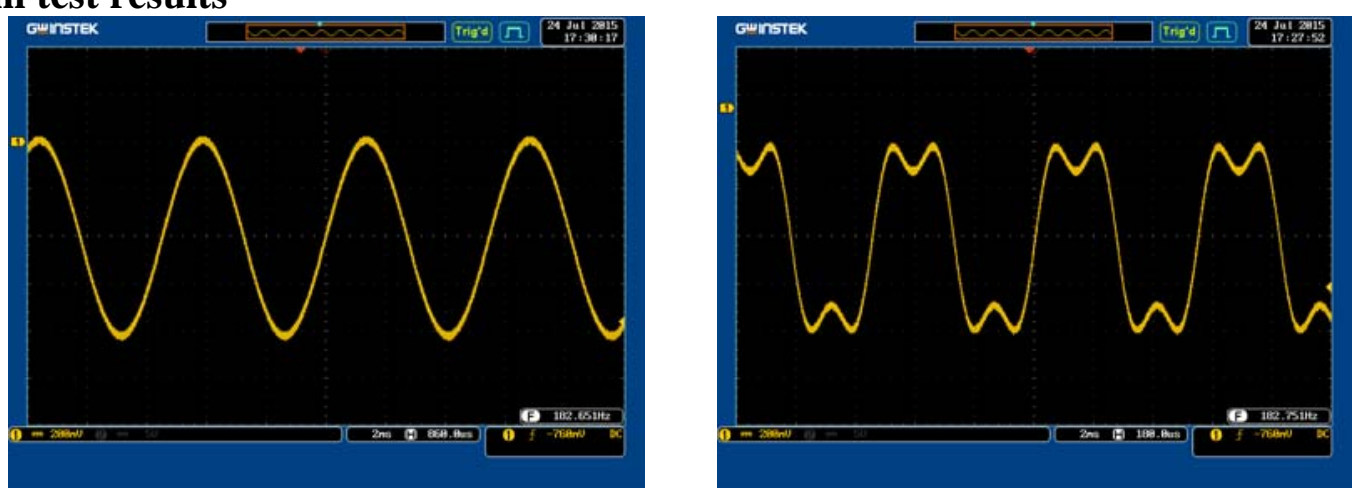

Fig. 9 A harmonic

Fig. 10 One, three times harmonic synthesis
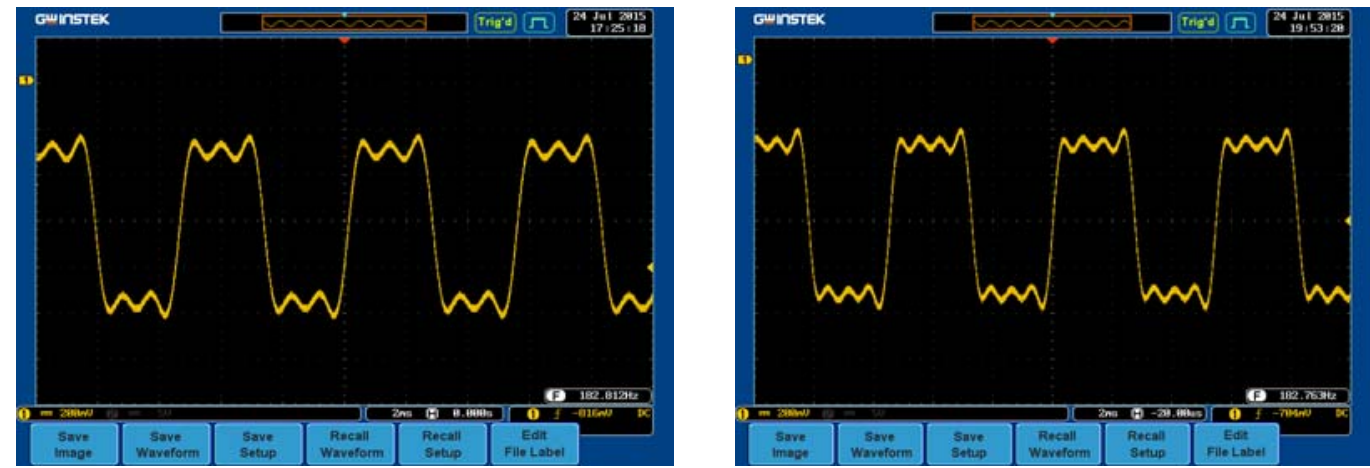

Fig. 11 One, three, five times harmonic synthesis

Fig. 12 One, three, five,seven times harmonic synthesis

Table 1 Four way signal for the synthesis of triangular waves

\begin{tabular}{|c|c|c|c|}
\hline frequency & peak & deviation & phase difference \\
\hline $200 \mathrm{~Hz}$ & $4.99 \mathrm{~V}$ & $2 \%$ & $0 \%$ \\
\hline $600 \mathrm{~Hz}$ & $1.65 \mathrm{~V}$ & $2.1 \%$ & $0.1 \%$ \\
\hline $993 \mathrm{~Hz}$ & $0.98 \mathrm{~V}$ & $4 \%$ & $0 \%$ \\
\hline $1.39 \mathrm{KHz}$ & $0.69 \mathrm{~V}$ & $3.38 \%$ & $0.2 \%$ \\
\hline \multicolumn{2}{|c|}{ peak of fang bo } & $4.98 \mathrm{~V}$ \\
\hline
\end{tabular}

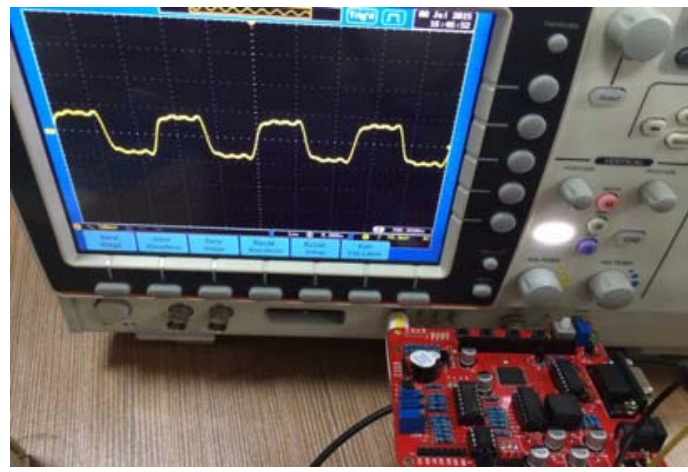

Fig. 13 Harmonic signal synthesis physical figure 


\section{Summary}

By comparison, we found the signal synthesis system designed by TLC5620 and STC15W1K24S can be very good to synthetic one, three, five, seven time's harmonic of the original square wave signal. Theoretical analysis was consistent with Matlab theoretical analysis. STC15W1K24S is faster than STC series microcontrolle, you can get stable base wave frequency up to $446 \mathrm{hz}$, which is more than ten times as much on STC series single-chip computer. The system can realize any four-way signal synthesis. At the same time, this system has low power consumption, amplitude phase control precision, operation convenient and reasonable cost, for constructing the concept of spectrum, the design has a good meaning, which has a certain promotion prospects.

\section{References}

[1] V. F. Kroupa. Phase and Amplitude Disturbances in Direct Digital Frequency Synth-esizers. IEEE International Frequency Control Symposium. (1999) No. 46, p. 481 - 486.

[2] Huang Yongan, Ma Lu, Liu Hui. MATLAB7.0/Simulink6.0 Modeling simulation development and advanced Engineering. Beijing: tsinghua university press, 2005, p. 100-125.

[3] Chen Shangsong, Lei Jia,Guo Qing. Electronic measurement and instrument.

Beijing: Electronic Industry Press, 2005, p. 89-105.

[4] Wang ao, Yi Bonian. Design and Implementation of Signal Waveform Synthesis Decomposition. Electric automatization, 2013, p. 66-70.

[5] Du Lei, Xue Zhongde, Ren Zhiguo. Design of interface between serial digital-to-analog converter chip TLC5620I and TMS320F2812.Electronic Design Engineering,2009,p.22-25.

[6] Zheng Runna,Hu Jianming,Hou Lijuan. The design and implementation of a signal generator Based on TLC5620.Beijing: Electronic measurement techniques, 2009, p.24-26. 